\title{
ON REAL QUADRATIC FIELDS OF CLASS NUMBER TWO
}

\author{
R. A. MOLLIN AND H. C. WILLIAMS
}

\begin{abstract}
It is the primary purpose of the paper to determine all real quadratic fields $Q(\sqrt{d})$ of class number $h(d)=2$ when $k \leq 24$ (with one possible exception). Here, $k$ is the period length of the continued fraction expansion of either $\omega=\sqrt{d}$, in the case $d \equiv 2$ or $3(\bmod 4)$, or of $\omega=(1+\sqrt{d}) / 2$, in the case $d \equiv 1(\bmod 4)$.
\end{abstract}

\section{INTRODUCTION}

In [6] the authors found all real quadratic fields $Q(\sqrt{d})$ with $h(d)=1$ and $k \leq 24, k$ as above, with one possible exception remaining. The result of [6] allowed a solution of several conjectures in the literature (see [3-6] for details). The techniques used there provide a basis for examining the class number 2 problem herein. We are able to improve upon them, and as a result, to reduce the computational workload for this paper. The new results (Theorem 2.1 and Lemmas 2.1 and 2.2) are of interest in their own right. In fact, Theorem 2.1 is a very useful means of immediately getting an explicit lower bound on the class number $h(d)$ in terms of $k$. The complete listings of our findings for $k \leq 24$ and $h(d)=2$ are in Tables 2.1 and 2.2 at the end of the paper. Moreover, our results vastly generalize the results of (and improve upon the techniques of) [9].

Throughout, $d$ will be a positive square-free integer. For convenience sake we give the basic continued fraction notation which we will use in the paper. For $\omega$ as in the abstract let the continued fraction expansion of $\omega$ be denoted by $\omega=\left\langle a, \overline{a_{1}, \ldots, a_{k}}\right\rangle$. Then $a_{0}=a=\lfloor\omega\rfloor$ and $a_{i}=\left\lfloor\left(P_{i}+\sqrt{d}\right) / Q_{i}\right\rfloor$ for $i \geq 1$ (here $\lfloor x\rfloor$ denotes the greatest integer less than or equal to $x$ ), where $\left(P_{0}, Q_{0}\right)=(\sigma-1, \sigma)$, with $\sigma=2$ if $d \equiv 1(\bmod 4)$ and $\sigma=1$ otherwise. Also, $P_{i+1}=a_{i} Q_{i}-P_{i}$ and $Q_{i+1} Q_{i}=d-P_{i+1}^{2}$ for $i \geq 0$. For more detailed information and connections with other topics, such as reduced ideals, the reader is referred to $[2,10]$.

\section{Class NUMBer 2 FOR $k<25$}

In order to find the real quadratic fields $Q(\sqrt{d})$ with $k \leq 24$ and $h(d)=2$, we proceed in a fashion similar to that in Mollin and Williams [6]. However,

Received by the editor November 30, 1989 and, in revised form, October 24, 1990 and November $20,1991$.

1991 Mathematics Subject Classification. Primary 11R11, 11R29.

Key words and phrases. Real quadratic field, class number, continued fraction.

(C) 1992 American Mathematical Society $0025-5718 / 92 \$ 1.00+\$ .25$ per page 
the techniques here are different in that we can reduce the amount of work by first proving the following result. This result is in fact an improvement on the inequality $R<k \log \sqrt{\Delta}$ used in [6] (here, $R=\log \varepsilon$, where $\varepsilon$ is the fundamental unit of $Q(\sqrt{d})$ ).

Theorem 2.1. If $R$ is the regulator of $Q(\sqrt{d})$, then $R<\lfloor 3(k+1) / 4\rfloor \log \sqrt{\Delta}$.

Proof. By results noted in Stephens and Williams [7] we have

$$
\varepsilon=\prod_{i=1}^{k} \varphi_{i}
$$

where $\varphi_{i}=\left(P_{i}+\sqrt{d}\right) / Q_{i}$ and $0<P_{i}<\sqrt{d}$. We can write $(2.1)$ as

$$
\varepsilon=\lambda \prod_{i=1}^{\lfloor k / 2\rfloor} \chi_{i}
$$

where $\chi_{i}=\varphi_{i} \varphi_{k-i+1}$ and

$$
\lambda= \begin{cases}1 & \text { when } 2 \mid k, \\ \varphi_{(k+1) / 2} & \text { when } 2 \nmid k .\end{cases}
$$

By [7, Theorem 2.1] we have $Q_{k-i}=Q_{i}$ and $P_{k-i}=P_{i+1}$; hence,

$$
\chi_{i}=\left(\left(P_{i}+\sqrt{d}\right) / Q_{i}\right)\left(\left(P_{i}+\sqrt{d}\right) / Q_{i-1}\right)=\left(\sqrt{d}+P_{i}\right) /\left(\sqrt{d}-P_{i}\right)
$$

from (2.2) of [7]. Furthermore, when $k$ is odd we have $Q_{(k-1) / 2}=Q_{(k+1) / 2}$, which means that $d=P_{(k+1) / 2}^{2}+Q_{(k+1) / 2}^{2}$ and therefore

$$
\lambda=\varphi_{(k+1) / 2}<\sqrt{\Delta}
$$

as $\sigma \mid Q_{i}$ for all $i \geq 0$.

If we define

$$
\nu=\left\{\begin{array}{l}
0 \text { when } 2 \mid k, \\
1 \quad \text { when } 2 \nmid k,
\end{array}\right.
$$

then it is an easy matter to show that

$$
\lfloor k / 2\rfloor+\lfloor(k+2) / 4\rfloor+\nu \leq\lfloor 3(k+1) / 4\rfloor \text {. }
$$

If $\sigma=2$ and $2\left\lfloor\lfloor\sqrt{d}\rfloor\right.$, then, since $P_{i} \equiv 1(\bmod 2)$ for all $i \geq 0$, we cannot have $P_{i}=\lfloor\sqrt{d}\rfloor$. Thus, in this case, $\chi_{i}<2 \sqrt{d}$ and $\varepsilon<\lambda(2 \sqrt{\Delta})^{\lfloor k / 2\rfloor}$, so

$$
\varepsilon<2^{\lfloor k / 2\rfloor}(\sqrt{\Delta})^{\lfloor k / 2\rfloor+\nu} \text {. }
$$

When $\Delta>16$, we have

$$
2^{\lfloor k / 2\rfloor}<(\sqrt{\Delta})^{\lfloor(k+2) / 4\rfloor},
$$

hence by (2.3) we have our result in this case. For the remaining values of $\Delta<16$ we see that $\sigma=2$ and $\lfloor\sqrt{d}\rfloor$ even forces $d=5$, for which the theorem is easily verified.

If $P_{i} \neq\lfloor\sqrt{d}\rfloor$ and $\lfloor\sqrt{d}\rfloor \equiv 1(\bmod \sigma)$, then we must have $P_{i} \leq\lfloor\sqrt{d}\rfloor-\sigma$ and $\chi_{i}<(2 / \sigma) \sqrt{d}=\sqrt{\Delta}$. Furthermore, if $j$ is the least positive integer such that $P_{j}=P_{j+1}$, we must have $k=2 j$. Therefore, the case in which we have the largest possible number of $P_{i}$-values equal to $\lfloor\sqrt{d}\rfloor$ can only occur when

$$
P_{1}=P_{2}=\cdots=P_{\lfloor(k+2) / 4\rfloor}=\lfloor\sqrt{d}\rfloor \text {. }
$$


Thus, if $n$ is the number of values of $P_{i}$ with $P_{i}=\lfloor\sqrt{d}\rfloor$ for $i \leq\lfloor k / 2\rfloor$, then

$$
\varepsilon<\lambda(\sqrt{\Delta})^{\lfloor k / 2\rfloor-n}(\sqrt{d}+\lfloor\sqrt{d}\rfloor) /(\sqrt{d}-\lfloor\sqrt{d}\rfloor)
$$

and $n \leq\lfloor(k+2) / 4\rfloor$.

Since $d-\lfloor\sqrt{d}\rfloor^{2} \geq \sigma^{2}$, we have $(\sqrt{d}+\lfloor\sqrt{d}\rfloor) /(\sqrt{d}-\lfloor\sqrt{d}\rfloor)<(2 \sqrt{d} / \sigma)^{2}=\Delta$. Hence,

$$
\varepsilon<\lambda(\sqrt{\Delta})^{\lfloor k / 2\rfloor-n} \Delta^{n}=(\sqrt{\Delta})^{\lfloor k / 2\rfloor+n+\nu} .
$$

By (2.3) the result follows.

We are now able to prove

Lemma 2.1. If $k \leq 24$ and $\Delta>6 \times 10^{9}$, then with at most one possible exception we must have $h(d)>2$.

Proof. By Tatuzawa [8], we have (with at most one exception) $L(1, \chi)>$ $0.655 \eta \Delta^{-\eta}$ for $0<\eta<\frac{1}{2}$ and $\Delta \geq \max \left(e^{1 / \eta}, e^{11 \cdot 2}\right.$ ) (where $L(1, \chi)=$ $\sum_{n=1}^{\infty}(\Delta / n) / n$ and $(\cdot / n)$ is the Kronecker symbol). Also, since $2 R h(d)=$ $\sqrt{\Delta} L(1, \chi)$, by Theorem 2.1 we must have

$$
2 h(d)>2 \Delta^{1 / 2-\eta}(0.655 \eta) /(\lfloor 3(k+1) / 4\rfloor \log \Delta)>4
$$

when $\Delta>6 \times 10^{9}, \eta=0.04442$, and $k \leq 24$.

In the case $\Delta=d \equiv 1(\bmod 4)$ we can improve Lemma 2.1 somewhat.

Lemma 2.2. If $d \equiv 1(\bmod 4), \Delta>4.75 \times 10^{9}$, and $k \leq 24$, then with at most one possible exception we must have $h(d)>2$.

Proof. This result can be easily verified by using the methods of Theorem 2.1 and Lemma 2.1 in the case where $\lfloor\sqrt{d}\rfloor$ is even. Thus, we will assume that $\lfloor\sqrt{d}\rfloor$ is odd and write $(2.5)$ as

$$
\varepsilon<(\sqrt{\Delta})^{\lfloor(k+1) / 2\rfloor-n}(\sqrt{d}+\lfloor\sqrt{d}\rfloor)^{n} \gamma^{-n},
$$

where $\gamma=\sqrt{d}-\lfloor\sqrt{d}\rfloor$. We also note that the value of $n$ (the number of values of $P_{i}=\lfloor\sqrt{d}\rfloor$ for $\left.i \leq\lfloor k / 2\rfloor\right)$ cannot exceed the number of divisors of $\left(d-\lfloor\sqrt{d}\rfloor^{2}\right) / 4$. This is a fact because each $Q_{i}$ associated with one of the $P_{i}$-values must be distinct from any other, must be even, and must be a divisor of $\left(d-\lfloor\sqrt{d}\rfloor^{2}\right) / 2$.

Now,

$$
\varepsilon<(\sqrt{\Delta})^{\lfloor(k+1) / 2\rfloor-n}(2 \sqrt{\Delta} / \gamma)^{n}=(\sqrt{\Delta})^{(k+1) / 2}(2 / \gamma)^{n}
$$

and

$$
R<\lfloor(k+1) / 2\rfloor \log \sqrt{\Delta}+n \log (2 / \gamma) .
$$

Hence, if $\Delta>4.75 \times 10^{9}, n \log (2 / \gamma)<52.6, \eta=0.045$, and $k \leq 24$, then

$$
2 h(d)>\Delta^{1 / 2-\eta}(0.655 \eta) /(\lfloor(k+1) / 2\rfloor \log \sqrt{\Delta}+n \log (2 / \gamma))>4 .
$$

If $n \log (2 / \gamma) \geq 52.6$, then, since $k \leq\lfloor(k+2) / 4\rfloor \leq 6$, we have $-\log \gamma>8.0$, $\gamma<0.000335$, and $d-\lfloor\sqrt{d}\rfloor^{2}<2 \sqrt{d} \gamma<46.2$. It follows that in this case $\left(d-\lfloor\sqrt{d}\rfloor^{2}\right) / 4<11$. However, the maximum value of the number of divisors of $l$ for $1 \leq l \leq 11$ is 4 , thus we must have $n \geq 4$. This now means that $-\log \gamma>12.4$ and $d-\lfloor\sqrt{d}\rfloor^{2}<1$, which is impossible. 
Thus, to find all real quadratic fields with $k \leq 24$ and $h(d)=2$ (with at most one more value remaining), we need only examine those with $d<1.5 \times 10^{9}$ when $d \not \equiv 1(\bmod 4)$ and those with $d<4.75 \times 10^{9}$ when $d \equiv 1(\bmod 4)$.

A computer search was run on all numbers of these forms up to the bounds given above to find all values of $d$ such that $k \leq 24$. Once this had been done, we used the method in Mollin and Williams [1] to eliminate most of the values of $d$ for which the corresponding field has $h(d)>2$. The value of $h(d)$ was actually determined for those fields which remained and those for which $h(d)>2$ were also eliminated, leaving only those for which $h(d)=2$. Our results are summarized in Tables 2.1 and 2.2. There were a surprisingly large number of them, 1958 to be exact.

TABLE 2.1. $h(d)=2$ for $k \leq 24$

\begin{tabular}{|c|c|}
\hline k & $\mathrm{d}$ \\
\hline 1 & $10,26,85,122,362,365,533,629,965,1685,1853,2813$ \\
\hline 2 & $\begin{array}{l}15,30,35,39,42,51,66,87,102,110,123,143,146,165,182,203,221,230,258,285, \\
327,357,402,447,635,645,678,741,843,902,957,1085,1245,1298,1517,1533,2037,2045 \\
2085,2397,2613,4245,4277,4773,5645,5957,6573,8333\end{array}$ \\
\hline 3 & $65,185,458,485,1157,2117,2285,3077,3293,3365,12365$ \\
\hline 4 & $\begin{array}{l}34,55,78,95,119,138,155,174,194,205,215,222,266,287,299,305,318,335,377,395,429, \\
482,527,623,755,782,861,885,1022,1055,1205,1405,1469,1965,2013,2093,2222,2301, \\
2373,2877,3005,3237,3597,3813,4893,5117,5397,5757,5885,6005,6285,6293,7157,7733, \\
7973,8357,9005,9077\end{array}$ \\
\hline 5 & $\begin{array}{l}74,218,493,565,1037,1565,1781,2138,2165,2173,3869,5165,5213,5837,6485,8021 \\
10397,14213\end{array}$ \\
\hline 6 & $\begin{array}{l}70,105,111,114,178,183,187,267,273,303,371,374,407,418,470,498,518,545,551,590, \\
602,618,642,803,805,822,923,1005,1007,1034,1118,1167,1173,1178,1202,1581,1605, \\
1623,1653,1707,1749,1790,2103,2109,2147,2245,2261,2445,2717,2723,2765,2845,3405, \\
3605,3638,3737,3893,4085,4301,4445,4605,5133,5453,7805,10237,10317,10653,11837 \\
12845,13253,13277,13445,14405,14573,15197,19445,21677,23693,25437\end{array}$ \\
\hline 7 & $\begin{array}{l}58,202,314,538,685,949,1165,1261,2885,3133,3277,3653,5429,5765,6437,7373,9197 \\
9509,12557,16757,17141,17261,18317,22301\end{array}$ \\
\hline 8 & $\begin{array}{l}91,238,282,638,695,707,710,854,866,942,1247,1403,1643,1655,1869,1883,1943,2238, \\
2390,2483,2685,2978,3205,3333,3765,4247,4565,5069,5141,5829,6341,6365,6693,6773, \\
6837,6965,7405,7469,8165,8853,9141,9453,9485,10013,10293,10373,10517,10797, \\
10805,11357,11501,15677,16805,17357,17853,19493,31533,37373,38213\end{array}$ \\
\hline 9 & $\begin{array}{l}106,698,1073,1189,1285,1385,1418,1865,2581,3233,4469,4553,4709,5597,8885,9365, \\
9773,9893,10229,10685,12053,12077,13565,14285,16733,23285,28757,29957\end{array}$ \\
\hline 10 & $\begin{array}{l}115,154,159,186,246,259,286,339,345,354,403,411,451,465,494,515,534,543,561,583, \\
591,598,665,671,682,687,703,705,762,779,830,938,978,1047,1102,1203,1263,1265, \\
1363,1383,1645,1671,1727,1742,2098,2123,2127,2485,2651,2658,2701,2747,2802,2829, \\
2867,2882,3157,3165,3218,3587,3685,3741,3743,3827,3867,4103,4619,4667,5057,5061,\end{array}$ \\
\hline & $\begin{array}{l}5205,5253,5285,5405,5522,6149,6613,6789,7005,7845,8045,8445,8517,8533,8621,9085, \\
9093,9581,9701,9821,10365,10645,10877,11373,11557,11973,12117,12165,12837, \\
14773,14861,16037,16077,16205,17045,17741,17877,18093,18357,18717,19253,21405, \\
21749,21885,22413,22517,22781,23933,23997,24213,24845,25077,25133,26333,26477, \\
27173,28005,28853,30245,30693,33677,37565,39245,41477,47195\end{array}$ \\
\hline
\end{tabular}


11

$265,298,554,794,1322,1658,2218,2509,3242,4181,4682,4685,11413,11773,13085,14453$, $15685,16085,18485,20285,20765,25565,28013,28685,31037,39797,40157,43733,46637$ 51917,56117

$247,295,355,366,385,386,426,535,609,767,802,815,851,969,995,1027,1113,1162,1207$, $1343,1353,1355,1358,1535,1538,1703,1717,1799,1910,1946,2018,2047,2054,2105,2231$, $2318,2327,2334,2365,2438,2507,2735,2855,2987,3002,3263,3302,3563,3695,4322,4382$, $4415,4453,4542,4717,4917,5447,6605,6853,6905,7365,7413,7797,9429,10262,11077$, $12341,12453,12485,12605,12669,13837,15333,16365,18557,18805,22893,23253,24293$, 24485,25397,25413,25517,27053,27389,27605,29141,29405,29861,30173,32357,36533, $40533,44117,44693,45485,45573,47157,52037,59213,59573,75677$

$746,778,1082,1241,1514,1649,2042,2426,3085,3338,3349,4058,4573,4589,4885,5389$, $7418,7421,8765,9389,9965,10085,12965,14837,16277,17533,19357,21053,22373,25877$, $30733,31373,31853,36965,38597,39437,40757,53477,69893,81413$

$190,319,406,430,471,474,611,667,670,699,742,745,806,807,1001,1043,1070,1115,1119$, $1309,1315,1338,1347,1398,1542,1545,1562,1634,1670,1691,1826,1839,1874,2282,2294$, $2315,2323,2337,2345,2427,2435,2463,2630,2714,2782,2821,3297,3378,3478,3621,3878$, $4115,4154,4178,4307,4331,4381,4499,4506,4646,4835,5222,5246,5282,5442,5673,5781$, $5917,6098,6213,6357,6443,6461,6477,6611,6645,7145,7285,7445,7619,7885,8205,8393$, $8437,8483,8565,8733,8805,8877,8965,9285,9645,9717,9877,10149,10573,11051,11805$, $12578,12621,12733,12869,12885,13197,13213,13973,14181,15861,15965,16541,17013$, $17805,18845,18941,19205,19277,19365,19397,19677,21197,21245,21549,21909,21917$, $22557,22965,23493,24069,24893,25109,25597,26285,26373,26885,27677,27845,28397$, $28605,28797,28805,31349,31413,32973,34013,34133,35045,35477,35765,35789,36917$, $38253,40445,42413,42933,43493,44957,45453,47253,51653,52013,54557,54677,55893$, $64181,64253,71357,85973,98045$

$481,1417,1466,2858,3065,3589,3785,3977,4538,5317,5941,6641,6749,7082,11861$, $12701,12833,13793,14909,16589,17153,18185,18365,18581,20885,24221,27989,29069$, $32885,33365,44813,47165,51173,66197,67973,70493,78917$

$310,391,415,654,655,679,955,1038,1146,1166,1267,1282,1346,1391,1578,1662,1739$, $1833,1858,1895,1902,2183,2195,2198,2407,2526,2553,2615,3227,3278,3374,3497,3565$, $3611,3755,3818,3918,4043,4069,4087,4142,4233,4298,4405,4955,4958,5123,5198,5267$, $5543,5558,5579,5726,5855,6062,6167,0254,6383,6501,6527,7322,7337,7355,7898,8029$, $8078,8207,8378,8421,8493,8718,9107,9309,9373,10509,11303,11405,11517,11917$, $12878,12957,13802,13943,15213,15365,15573,16685,17673,17997,19293,19389,21965$, $22029,22173,24101,25885,26133,29685,30413,30581,31493,34989,35861,38309,38405$, $38517,40685,41741,43053,43253,43805,45773,48965,49565,49685,50973,55013,55173$, $57293,58253,58373,58973,59237,61277,63557,67133,67205,67997,69621,75413$

$1018,1994,2965,4285,5354,5498,5585,8917,9242,9665,10265,12085,13061,13957$, $14677,15242,15613,16109,16565,16613,17173,17285,17429,17861,18037,18737$, $18965,34037,34957,35285,36413,37949,40085,40501,41165,47813,48149,50357,53285$, $57797,58853,61133,62957,63653,86957,146453$

$519,562,831,879,951,1185,1199,1209,1281,1310,1362,1379,1505,1506,1526,1606,1630$, $1686,1698,1842,1903,1919,1923,1983,1991,2202,2219,2283,2363,2631,2697,2771,2985$, $3183,3282,3414,3470,3642,3702,3707,3830,3839,4029,4287,4343,4430,4562,4697,4791$, $4803,5027,5363,5705,5797,5845,5870,6177,6182,6278,6407,6470,6758,6767,6830,6842$, $7358,7485,7802,7869,7958,8582,8589,8697,8843,9119,9269,9381,9383,9445,9470$, $10245,10461,10502,10643,11397,12093,12162,12278,12549,12722,12749,12909,13019$, $13237,13593,13605,13682,13821,14053,14309,14605,15485,15933,16845,17197,17381$, $17733,18173,19229,19245,20253,20541,20645,20677,20917,21533,22085,22181,22245$, 23309,24357,24365,24477,24837,25445,25485,25781,26781,27485,28461,28589,29309, 30317,30957,31733,32021,32669,32773,33789,33845,34405,34685,34853,35189,36669, $36813,39893,40613,41789,41837,41973,43581,43589,43797,44645,47333,47549,48245$, $49805,50133,51557,51845,52853,54197,54845,57845,59637,61477,62405,64277,66549$, $70133,71213,73253,74973,76037,80013,80117,92477,96701,128117,138773,139277$, $146333,151373,168773,171797$

$922,1706,2186,2257,3386,8522,8714,9997,16781,17177,20513,20813,21509,24341$, $26165,28453,29597,30365,31085,35333,35885,36173,37685,37757,38765,41765,43469$, $46157,50453,52637,53765,57965,59765,62285,65501,70733,75197,79085,82277,84773$, $107333,109757,139037,144317$ 
TABLE 2.1. (continued)

$511,559,606,790,1002,1065,1079,1182,1195,1374,1415,1510,1513,1537,1603,1687$, $1961,2193,2215,2455,2471,2627,2863,3155,3239,3295,3383,3647,3746,3857,4163,4295$, $4458,4535,4595,4727,4782,4847,4922,5038,5143,5195,5258,5678,5709,5759,5803,5822$, $5962,6107,6141,6338,6415,6467,6702,6914,6943,7189,7295,7343,7367,7787,7813,7895$, $8238,8258,8507,8567,8903,9527,9861,9885,10622,11949,13413,13461,14541,14565$, $15029,15203,16237,16463,16989,18821,19085,19221,19337,19509,19533,20517,20642$, $21093,21765,21962,23069,23133,25197,25557,26637,26697,28205,28965,29261,29365$, $32053,32997,34773,35085,36093,36165,36237,36573,38685,39093,40557,41285,42605$, $42749,43085,44765,45933,46277,48237,48453,49181,50213,52917,54893,57189,58493$, $58533,60205,61053,61773,61973,62237,62933,63213,65285,65813,67373,69293,70213$, $71405,74261,76677,77957,80693,80717,81317,81357,81437,82869,88413,93197,96773$, $106685,109997,113693,115037,240077$

$394,865,1769,1985,2561,2762,3098,4385,5465,5485,5965,6122,7141,7265,10565,11101$, $11485,11581,13285,13466,14381,14765,16442,21365,22565,28373,34493,35197,36221$, $44861,47477,48485,48941,51365,54317,57317,58133,58589,69365,75917,78053,78557$, $78773,80165,84173,85277,85949,89333,91013,92165,94877,97877,104837,120893$, $127613,130037,156917,167477,212357$

$466,763,771,834,1059,1194,1266,1334,1558,1563,1798,1835,1843,1905,1963,1986$, $2001,2082,2270,2274,2279,2406,2514,2519,2546,2585,2643,2778,2823,2859,2931,2937$, $2947,3063,3107,3131,3147,3182,3207,3310,3417,3506,3635,3657,3687,3938,4119,4145$, $4187,4202,4433,4630,4645,4814,4863,4883,4938,4965,5111,5163,5315,5345,5367,5603$, $5703,5718,5747,5862,5989,6023,6061,6378,6387,6403,6431,6585,6635,6738,6743,7026$, $7122,7143,7257,7334,7545,7553,7622,7701,7842,7982,8018,8027,8270,8365,8531,8630$, $8749,8897,8998,9113,9138,9158,9205,9263,9687,9709,10190,10298,10307,11507$, $11747,12257,12261,12305,12405,12662,12827,13067,13265,13405,13817,14205,14510$, $14845,15085,15113,15617,15765,16149,16797,17445,17549,18002,20301,21837,23777$, $24141,24405,25149,25653,25682,26197,27101,28673,29805,30165,31965,32469,33045$, $33429,33549,33645,33989,34005,34933,36581,37077,37317,37445,37677,39693,40605$, 42117,44373,45237,45605,46949,48045,48813,48885,49629,49677,50045,50861,51861, $52085,52421,54645,56069,56357,56397,57749,57893,58197,58205,59285,60277,62733$, $63237,63437,63453,64085,65189,66557,67469,68933,71021,71885,72597,76053,76773$, $77357,77693,78869,80597,83765,85917,86933,89957,92573,94085,94317,95933,98813$, $99413,101213,101909,104357,106757,107645,112013,116045,116261,116597,120245$, $121733,126245,130613,135453,136037,136253,138437,145277,145613,148133,148973$, $149357,149957,150293,156053,157493,165557,168797,204245,248093$

$586,634,1585,2474,3578,4121,4141,5114,6074,6109,6362,6506,6602,7261,8042,8249$ $10673,12349,20557,22837,24869,26773,26869,33017,34165,34541,37661,37837,43693$, $51757,55565,56285,56381,58277,59293,63677,64349,67253,74693,76565,77453,86213$, $87485,90485,90557,90653,94973,99557,107117,107573,107957,113237,119477,139157$, $154853,160277,172133,176837,247397$

$826,871,1147,1255,1614,1711,1795,2051,2119,2154,2409,2414,2534,2594,2698,2743$, $2759,3009,3018,3110,3206,3633,3806,3854,3882,4031,4118,4310,4638,4665,4826,5322$, $5466,6155,6302,6455,6618,7091,7222,7278,7763,8302,8489,8927,8939,9002,9287,9347$, $9393,9469,9741,9785,10058,10142,10415,10442,10562,10823,11042,11262,11546$, $11714,11843,12311,12741,13118,13502,13958,14198,14987,15815,16502,16765,16859$, $17063,17447,17501,17531,18381,18501,18885,19685,20013,21047,21287,21565,21669$, $22523,23037,23927,25053,25322,26277,27069,27669,27789,27853,29877,30093,31485$,

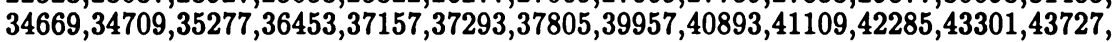
$44133,44429,44717,45069,47613,48005,48765,49101,50165,51837,54389,54797,54885$, $56885,57813,59717,59853,60477,61269,61365,62493,63885,68285,70565,70685,71133$, $71165,72197,72917,76973,78837,83613,84413,85533,87989,88877,89549,91077,92405$, $92597,97277,102605,106413,109205,109805,112517,113813,118685,122405,125333$, $126965,132117,136205,141797,151205,154013,158933,165413,169133,172493,175637$, 197333,205805 
TABLE 2.2

\begin{tabular}{|c|c|}
\hline$k$ & The number of $h(d)=2$ \\
\hline 1 & 12 \\
\hline 2 & 48 \\
\hline 3 & 11 \\
\hline 4 & 58 \\
\hline 5 & 18 \\
\hline 6 & 79 \\
\hline 7 & 24 \\
\hline 8 & 59 \\
\hline 9 & 28 \\
\hline 10 & 135 \\
\hline 11 & 31 \\
\hline 12 & 102 \\
\hline 13 & 40 \\
\hline 14 & 169 \\
\hline 15 & 37 \\
\hline 16 & 130 \\
\hline 17 & 46 \\
\hline 18 & 187 \\
\hline 19 & 44 \\
\hline 20 & 161 \\
\hline 21 & 59 \\
\hline 22 & 245 \\
\hline 23 & 59 \\
\hline 24 & 176 \\
\hline
\end{tabular}

\section{ACKNOWLEDGMENTS}

The first author's research was supported by NSERC Canada grant \#A8484; and that of the second author by NSERC Canada grant \#A7649. Also the authors wish to thank Gilbert Fung, a graduate student of the second author, for doing the computing involved in Tables 2.1 and 2.2.

\section{BIBLIOGRAPHY}

1. R. A. Mollin and H. C. Williams, On a solution of a class number two problem for a family of real quadratic fields, Computational Number Theory (A. Pethö, M. Pohst, H. Williams, and H. Zimmer, eds.), Walter de Gruyter, Berlin, 1991, pp. 95-101.

2. _ Computation of the class numbers of a real quadratic field, Advances in the Theory of Computing and Comput. Math. (to appear). 
3. R. A. Mollin and H. C. Williams, Prime-producing quadratic polynomials and real quadratic fields of class number one, Number Theory (J. M. DeKoninck and C. Levesque, eds.), Walter de Gruyter, Berlin, 1989, pp. 654-663.

4. _ Class number one for real quadratic fields, continued fractions and reduced ideals, Number Theory and Applications (R. A. Mollin, ed.), Kluwer, Dordrecht, 1989, pp. 481496.

5. __ Solution of the class number one problem for real quadratic fields of extended RichaudDegert type (with one possible exception), Number Theory (R. A. Mollin, ed.), Walter de Gruyter, Berlin, 1990, pp. 417-425.

6. - On a determination of real quadratic fields of class number one and related continued fraction period length less than 25, Proc. Japan Acad. Ser. A Math. Sci. 67 (1991), 20-25.

7. A. J. Stephens and H. C. Williams, Some computational results on a problem concerning powerful numbers, Math. Comp. 50 (1988), 619-632.

8. T. Tatuzawa, On a theorem of Siegel, Japan J. Math. 21 (1951), 163-178.

9. H. Taya and N. Terai, Determination of certain real quadratic fields with class number two, Proc. Japan Acad. Ser. A Math. Sci. 67 (1991), 139-144.

10. H. C. Williams and M. C. Wunderlich, On the parallel generation of the residues for the continued fraction factoring algorithm, Math. Comp. 48 (1987), 405-423.

Department of Mathematics, University of Calgary, Calgary, Alberta T2N 1N4, CANADA

E-mail address: ramollin@acs.ucalgary.ca

Department of Computer Science, University of Manitoba, Winnipeg, Manitoba R3T 2N2, CANADA

E-mail address: Hugh_Williams@csmail.cs.umanitoba.ca 